\title{
Clinicopathologic Features and Outcome of Adenocarcinoma of the Anal Canal: A Population-Based Study
}

\author{
Shekhar Gogna, Roberto Bergamaschi, Agon Kajmolli, Mahir Gachabayov ${ }^{\mathbb{D}}$, Aram Rojas, \\ David Samson, Rifat Latifi $\mathbb{D}$, and Xiang Da Dong $\mathbb{D}$
}

Division of Surgical Oncology, Westchester Medical Center, New York Medical College, 100 Woods Road, Taylor Pavilion, Office Suite \#353, Valhalla, NY 10595, USA

Correspondence should be addressed to Xiang Da Dong; xddong@hotmail.com

Received 16 March 2020; Accepted 21 April 2020; Published 13 May 2020

Academic Editor: Vahit Ozmen

Copyright ( $) 2020$ Shekhar Gogna et al. This is an open access article distributed under the Creative Commons Attribution License, which permits unrestricted use, distribution, and reproduction in any medium, provided the original work is properly cited.

Background. Anal canal adenocarcinoma (AA) is an uncommon tumor of the gastrointestinal tract. We seek to provide a detailed description of the incidence, demographics, and outcome of this rare tumor in the United States. Methods. The data on anal canal adenocarcinoma from SEER Program, between 1973-2015, were extracted. We analyzed the incidence rates by demographics and tumor characteristics, followed by analysis of its impact on survival. Results. The incidence of AA increased initially by $4.03 \%$ yearly from 1973 to 1985 but had a modest decline of $0.32 \%$ annually thereafter. The mean age for diagnosis of AA was $68.12 \pm 14.02$ years. Males outnumbered females by 54.8 to $45.2 \%$. Tumors were mostly localized on presentation (44.4\%) and moderately differentiated (41.1\%). Age generally correlated with poor overall cancer survival. However, young patients (age $<40$ years) also showed poor long-term survival. Patients with localized disease and well-differentiated tumors showed better survival outcomes. Surgical intervention improved survival significantly as compared to patients who did not (116.7 months vs 42.7 months, $p<0.01)$. Conclusions. Anal canal adenocarcinoma demonstrated a poor bimodal cancer-free survival in both younger and older patient groups. Surgery significantly improves odds of survival and should be offered to patients amenable to intervention.

\section{Introduction}

Adenocarcinoma of the anal canal is a rare neoplasm. Worldwide, the incidence is only a few thousand cases per year. Histologically, it represents approximately $16.5 \%$ of all types of anal canal cancers, which is dominated by squamous cell carcinoma [1]. The anal canal extends from the anal margin to the anorectal ring/flexure representing the terminal part of the gastrointestinal tract. Anatomically, based on the lining epithelium, the anal canal can be divided into the colorectal zone defined by the colorectal type of glandular mucosa proximally, the anal transition zone defined by the variable appearance of the lining epithelium in the middle, and the distal portion lined by squamous epithelium [1, 2].

Several proposals have been made as to the pathologic mechanisms leading to the anal canal adenocarcinomas (AA). These include anal glandular carcinomas originating from the anal glands, colloid carcinomas associated with Paget's disease of the anus, and adenocarcinomas arising from chronic fistula and inflammatory epithelium in the anus, as well as adenocarcinomas that arose from the distal rectum with extension into the anal canal [3, 4]. Previous observations that these malignancies were associated with chronic intestinal diseases such as preexisting fistulas or Crohn's disease prompted the hypothesis for the pathologic development of adenocarcinoma of the anal canal [3].

More recently, mutational analysis was able to differentiate anal canal adenocarcinoma into region-specific subtypes. The differences in HPV (16 and 18) infection status and expression of the immune checkpoint and mutational profile of several targetable genes separate this neoplasm into the 2 distinct entities: anal glandular/transitional subtype and colorectal subtype. From a treatment standpoint, anal glandular/transitional type cancers respond poorly to 
standard treatments. Mutational analysis showed that it harbored less frequent mutations in downstream factors of the EGFR signaling pathway, but a significantly higher expression of immune checkpoint inhibitors PD1/PD-L1 compared to its colorectal subtype counterpart. These tumors are Krt7 positive as opposed to Krt20 and CDX2 positive commonly seen with the colorectal subtype. The other colorectal subtype appears to be closely related to the tumors arising from the colorectal mucosa [5].

Anal canal adenocarcinomas are frequently thought to be more aggressive than squamous cell carcinomas [6, 7]. Traditional management of adenocarcinomas of the anal canal has relied on multimodal treatment to avoid local or distant failures. Currently, combined multimodality with radical surgical resection appears to portend more favorable prognosis [8]. Fiveyear overall survival is thought to exceed $60 \%$ following curative surgery in combination with chemoradiation $[8,9]$.

Because of the location of the tumor, the pattern of recurrence also differs from traditional rectal cancer [10]. Patients tend to have a higher incidence of lymph node metastasis in the groins necessitating concurrent management of the inguinal canal. With the recent advent of personalized genomic medicine, we seek to evaluate the epidemiology and overall prognosis of patients afflicted with this disease based on the large SEER database and hopes to provide insight on the patterns of treatment and failures.

\section{Materials and Methods}

This is a retrospective cohort study from the SEER database using 18 registries to identify all patients with anal canal adenocarcinoma from 1973-2015, using SEER site specific primary code, based on the International Classification of Diseases for Oncology, third edition (ICD-O-3). Anal canal cancers were classified by site: anal, not otherwise specified (NOS), (C21.0), anal canal (C21.1), and cloacogenic zone (C21.2). Patients with adenocarcinoma of anal canal overlapping with the rectum (C21.8) were excluded, as there is significant heterogeneity and difficulty to distinguish from patients with low lying rectal cancers. The histology codes used for adenocarcinoma were extracted from ICD-O-3 and are listed in Supplementary Table S1. The histological subtypes of various types of adenocarcinoma are listed in Supplementary Table S2.

2.1. Statistical Analysis. We used the frequency and survival session from SEER * Stat version 8.3.5 to collect incidence, trend analysis, and 1 through 5-year survivals based on age, gender, location, histology, and malignant behavior. Patients with missing data were excluded from the analysis. Statistical analysis was conducted using SPSS for IBM Corp. (IBM SPSS Statistics for Windows, Released 2017, Version 25.0. Armonk, NY: IBM Corp). The overall survival (OS) rates were calculated using the actuarial (Kaplan-Meier) method. Differences in survival based on age, gender, histology, grade, and surgery were computed using a log rank test (Mantzel-Cox). Multivariate analysis was conducted using Cox regression analysis to identify the independent effect of cancer type on survival controlling for age, year of diagnosis, gender, race, tumor stage, and grade. All $p$ values were 2sided and the $p$ value of $<0.05$ was considered significant.

According to the hospital policy, the institutional review board approved and exempted the study. This retrospective observational study is reported according to STROBE guidelines [11].

\section{Results}

There were 2090 patients with anal canal adenocarcinoma (AA). The median age for AA was 68.12 \pm 14.02 years (range was 20-100 years). The disease showed male $(n=1145,54.8 \%)$ preponderance as compared to females $(n=945,45.2 \%)$. The disease, without normalizing to population demographics, was more commonly reported in whites $(n=1629,77.9 \%)$, followed by blacks $(n=291,13.9 \%)$ and all others $(n=170,8.1 \%)$. Tumor grade ranged from Grade I well differentiated $(n=263,12.6 \%)$, grade II moderately differentiated $(n=858,41.1 \%)$, and grade III poorly differentiated $(n=340,16.3 \%)$ to grade IV undifferentiated $(n=31,1.5 \%)$ and cell type not determined $(n=598$, $28.6 \%$ ) (Supplementary Table S2). Based on the SEER database, the extent of disease was in situ $(n=141,6.7 \%)$, localized $(n=927,44.4 \%)$, regional $(n=539,25.8 \%)$, and distant $(n=281$, $13.5 \%)$, as well as incomplete information $(n=201,9.6 \%)$.

3.1. Incidence Rates. The estimated current prevalence rate of adenocarcinoma of anal canal is $0.0011 \%$ as calculated from $\mathrm{SEER} * \mathrm{Stat}$. The annual percentage change (APC) is used to measure trends or the change in rates over time. The Joinpoint software 6.0.0 was used to calculate the annual percentage change (APC) in incidence rates from 1973 to 2015. The incidence of AA increased annually by $4.03 \%$ from 1973 to 1985 and it showed a downward trend annually by $0.32 \%$ from 1986 to 2015 . This rise and then decrease in annual incidence rate are shown in Figure 1. The reasons for this significant change in epidemiology remain to be investigated.

3.2. Survival. We stratified the patients into four age groups ( $<40$ years, $41-60$ years, $61-80$ years, and $\geq 81$ years). Elderly patients $>81$ years had the worst survival (mean survival of $30.14 \pm 1.84$ months $) \quad(\mathrm{HR}, 3.79 ;$ 95\% CI, 2.65-5.41) $(p<0.01)]$, as shown by the Kaplan-Meier Plot (Figure 2). Interestingly, patients who were younger than 40 years and elderly patients aged $>81$ years both showed initial poor cancer-specific survival rates. Patients in group II (41-60 years) had the best 1 through 5 -year cancer-specific survival rate followed by patients in group III (61-80 years) (Figure 3). Eventually, group 1 patients (<40 years) showed improved long-term disease-free survival after 5 years. The calculated overall 1-, 2-, 3-, 4-, and 5-year survival rates are $76.1 \%, 63.4 \%, 52.6 \%, 47.9 \%$, and $39.6 \%$, respectively. Amongst patients with AA, males outnumbered females ( 54.8 vs $45.2 \%)$. The survival in males as compared to females was 98.8 vs 88.6 months, respectively, which was not statistically significant $(p=0.13)$. Anal canal adenocarcinoma was also more common in whites (77.9\%), although not normalized to the population. However, race was also 


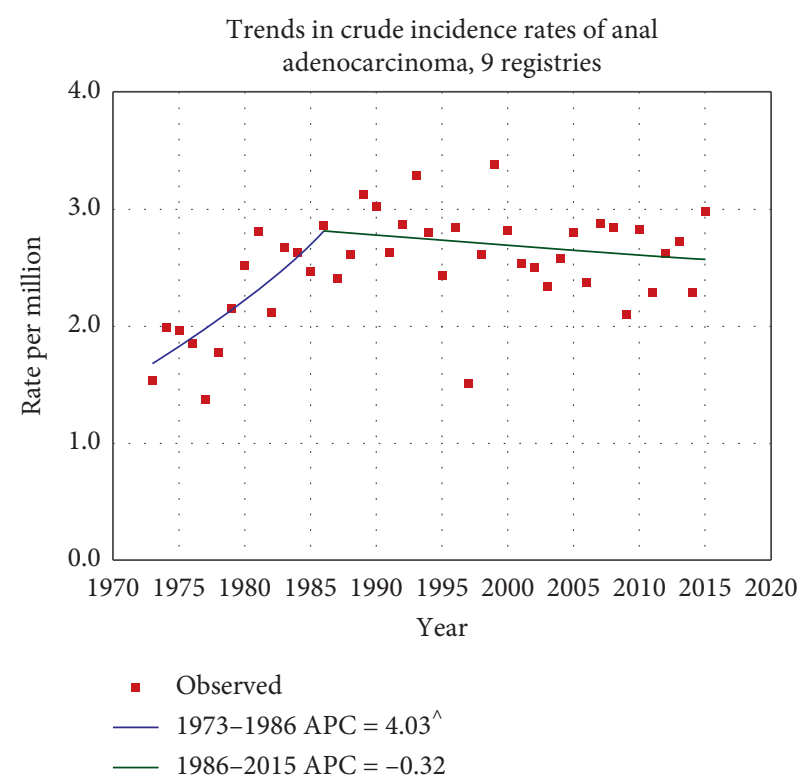

${ }^{\wedge}$ Indicates that the annual percent change (APC) is significantly different from zero at the alpha $=0.05$ level. Final selected model: 1 joinpoint.

FIgURE 1: Changing incidence from 1973-2015.

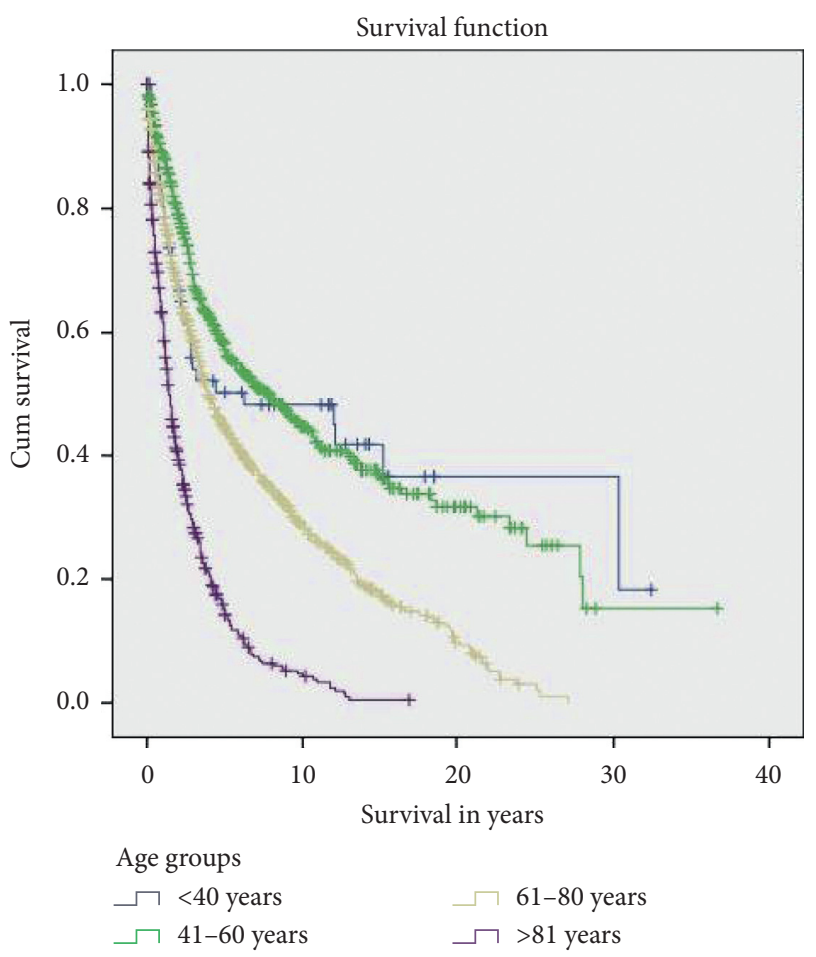

FIGURE 2: Kaplan-Meier plots showing overall survival among patients with anal canal adenocarcinoma (AA) based on age groups.

not a statistically significant factor $(p=0.06)$ influencing survival.

The primary site for anal canal cancer is divided into three zones according to ICD-O-3 morphology codes: anal,

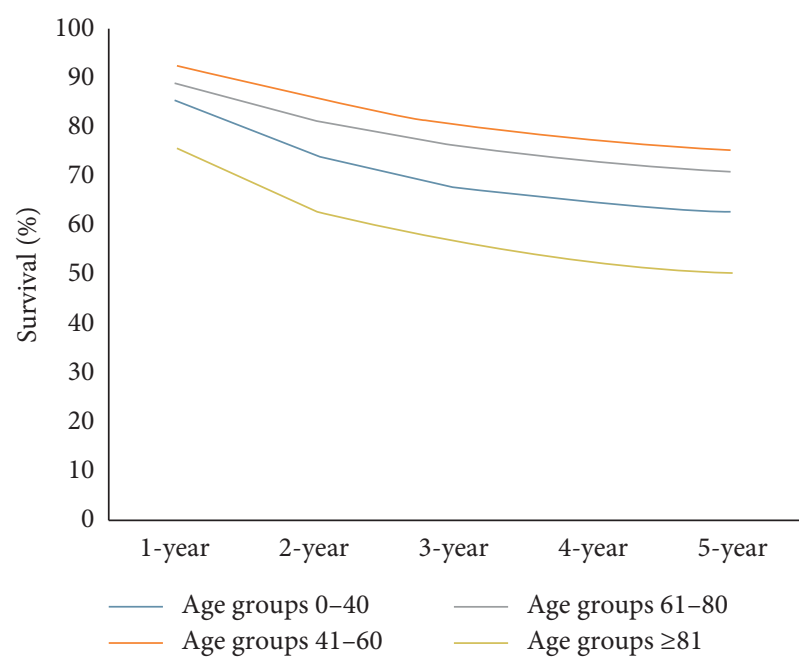

Figure 3: Trend analysis of cancer-free survival rates according to various age groups.

not otherwise specified (NOS), anal canal, and cloacogenic zone. We excluded cancers of anal canal overlapping with the rectum. The mean survival was highest in cancer of the anal canal with mean survival being $98.38 \pm 5.56$ months. However, location of cancer did not have statistical significance on long-term survival $(p=0.405)$.

Anal canal adenocarcinoma had 12 various subtypes (not all listed); adenocarcinoma NOS (the general variety) was the most common subtype (Supplementary Table S2). The survival was best when AA originated in the polyps $(172.90 \pm 15.25$ months), which was statistically significant $(p<0.01)$. This emphasizes the importance of carefully screening for the polyps in the anal canal.

As expected, the survival is best in patients who present with localized disease (mean survival in months $166.88 \pm 12.62$ ) as compared to patients presenting with distant metastasis (33.81 \pm 6.024 months). Patients with metastatic disease have 6fold mortality rate (HR, 6.02; CI, 4.55-7.99; $p<0.0001$ ). The survival curve in Figure 4 shows that as with increasing stage of the disease, overall survival decreased. The survival characteristics are summarized in Table 1.

Table 2 shows the results of a multivariate Cox regression analysis among patients with AA to study the effect of various patient-related factors on survival. Increasing age (HR, 3.79; 95\% CI, 2.65-5.41; $p<0.01$ ), advanced stage of the disease (regional disease HR, 1.27; 95\% CI, 1.08-1.49; $p<0.01$ ) (distant disease HR, 2.8; 95\% CI, 2.35-3.36; $p<0.01)$, and no surgical intervention $(p<0.01)$ significantly influenced the survival among patients with AA. There was a strong trend of improved survival among patients who received surgery.

\section{Discussion}

Anal canal adenocarcinoma is an uncommon diagnosis that portends poor overall survival [12]. The majority of anal canal cancers are squamous cell type carcinomas which are currently treated with chemoradiation [13]. Therefore, the management of AA is not standardized and fragmented in 


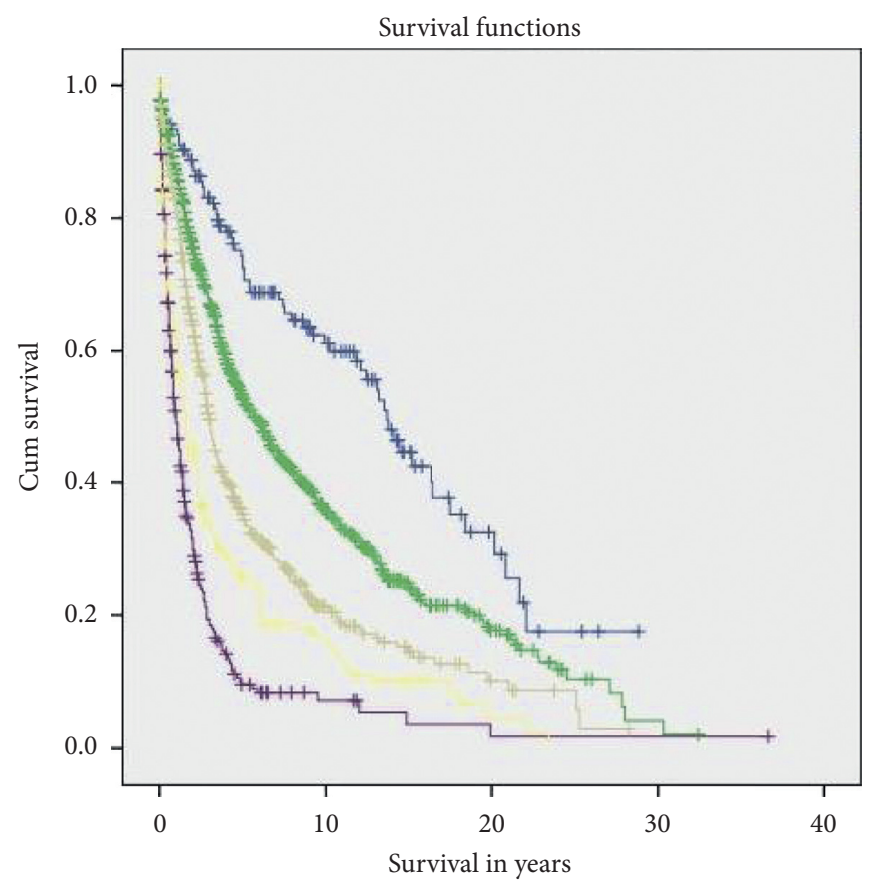

SEER historic stage

$\begin{array}{lll}\neg \text { In situ } & \neg \text { Distant } \\ \neg \text { Localized } & \text { Unstaged } \\ \square & \text { Regional } & \end{array}$

FIGURE 4: Kaplan-Meier plots showing overall survival among patients with anal canal adenocarcinoma (AA) based on pathologic stage.

TABLE 1: Survival characteristics of patients with anal canal adenocarcinoma.

\begin{tabular}{|c|c|c|c|c|}
\hline Variable & Survival (months) & SD & $95 \% \mathrm{CI}$ & $p$ value \\
\hline \multicolumn{5}{|l|}{ Age } \\
\hline$<40$ years & 167.55 & 24.13 & $120.1-214.9$ & \multirow{4}{*}{$<0.01$} \\
\hline $41-60$ years & 162.85 & 11.00 & $141.2-184.4$ & \\
\hline $61-80$ years & 86.75 & 3.33 & $80.2-93.2$ & \\
\hline$\geq 81$ years & 30.14 & 1.84 & $26.5-33.7$ & \\
\hline \multicolumn{5}{|l|}{ Gender } \\
\hline Male & 88.60 & 4.71 & $79.3-97.8$ & \multirow{2}{*}{0.13} \\
\hline Female & 98.84 & 4.62 & $89.7-107.9$ & \\
\hline \multicolumn{5}{|l|}{ Race } \\
\hline Whites & 94.70 & 4.08 & $86.6-102.7$ & \multirow{4}{*}{0.06} \\
\hline African Americans & 78.66 & 6.99 & $64.9-92.3$ & \\
\hline Asians/Pacific & 131.03 & 29.75 & $72.7-189.3$ & \\
\hline Others & 104.08 & 10.38 & $83.7-124.4$ & \\
\hline \multicolumn{5}{|l|}{ Stage } \\
\hline In situ (0) & 166.88 & 12.62 & $142.1-191.6$ & \multirow{5}{*}{$<0.01$} \\
\hline Localized (1) & 114.29 & 4.92 & $104.6-123.9$ & \\
\hline Regional (2) & 77.26 & 5.26 & $66.9-87.5$ & \\
\hline Distant $(3)$ & 33.18 & 6.02 & $22.0-45.6$ & \\
\hline Information not sufficient (9) & 51.63 & 5.80 & $40.2-63.0$ & \\
\hline \multicolumn{5}{|l|}{ Surgery } \\
\hline Yes & 116.74 & 4.58 & $107.7-125.7$ & \multirow{2}{*}{$<0.01$} \\
\hline No & 42.70 & 2.91 & $36.9-48.4$ & \\
\hline
\end{tabular}

practice until recently, as proposed by NCCN guidelines [1]. Because of the rarity of this disease, most reports in the literature consisted of case series or case reports that are described in a retrospective fashion. Limited long-term follow-up data are available with regards to this unusual diagnosis. Because of the unfavorable outcome seen with this 
TABLE 2: Cox hazard regression of predictors of survival.

\begin{tabular}{|c|c|c|c|}
\hline Variable & HR & $95 \%$ of HR & $p$ value \\
\hline \multicolumn{4}{|l|}{ Age } \\
\hline $0-40$ years (reference) & 1 & & \\
\hline $41-60$ years & 0.97 & $0.67-1.38$ & 0.866 \\
\hline $61-80$ years & 1.62 & $1.15-2.30$ & 0.006 \\
\hline$>81$ years & 3.79 & $2.65-5.41$ & $<0.01$ \\
\hline \multicolumn{4}{|l|}{ Race } \\
\hline American Indians/Alaska Natives (reference) & 1 & & \\
\hline Whites & 1.42 & $0.80-2.56$ & 0.21 \\
\hline African Americans & 1.62 & $0.90-2.90$ & 0.29 \\
\hline Asians/Pacific Islanders & 1.19 & $0.60-2.198$ & 0.30 \\
\hline \multicolumn{4}{|l|}{ Gender } \\
\hline Female (reference) & 1 & & \\
\hline Male & 1.08 & $0.97-1.20$ & 0.13 \\
\hline \multicolumn{4}{|l|}{ Stage } \\
\hline In situ (reference) & 1 & & \\
\hline Localized only & 0.87 & $0.75-1.00$ & 0.06 \\
\hline Regional spread by direct extension only & 1.27 & $1.08-1.49$ & 0.03 \\
\hline Distant site(s)/node(s) involved & 2.8 & $2.35-3.36$ & $<0.01$ \\
\hline Unknown & 2.1 & $1.76-2.76$ & $<0.01$ \\
\hline \multicolumn{4}{|l|}{ Grade } \\
\hline Well differentiated (reference) & 1 & & \\
\hline Moderately differentiated; & 1.37 & $1.15-1.64$ & $<0.01$ \\
\hline Poorly differentiated; differentiated & 2.15 & $1.76-2.63$ & $<0.01$ \\
\hline Undifferentiated; anaplastic & 1.81 & $1.37-2.88$ & 0.01 \\
\hline Cell type not determined & 1.11 & $0.92-1.34$ & 0.24 \\
\hline \multicolumn{4}{|l|}{ Surgery } \\
\hline Yes (reference) & 1 & & \\
\hline No & 0.38 & $0.34-0.42$ & $<0.01$ \\
\hline
\end{tabular}

disease, there has been studies in the past suggesting that chemoradiation should be the only treatment option $[14,15]$. Initial proposal by Papagikos et al. modelled the treatment similar to management of squamous cell carcinoma of the anal canal, albeit with poor outcomes with 5year survival at $19 \%$ [14]. Subsequent studies from the MD Anderson and Memorial Sloan Kettering Cancer Center showed that combined modality including surgery can improve 5-year survival $[9,16]$. These are some of the data that led to NCCN proposing a combined approach for management of this rare cancer in 2004.

There remains a lot of controversy in terms of management of this disease over the last decade. Belkacemi et al. analyzed the Rare Cancer Network and showed a respectable 5 -year overall survival rate of $58 \%$ for patients treated with chemoradiation alone [15]. In contrast, a previous analysis of the SEER database by Franklin et al. concluded that those undergoing surgery showed improved survival compared with no surgical intervention [17]. However, some case series have shown that aggressive surgical resection along with chemoradiation may offer chances of long-term improved survival [8]. Li et al. analyzed the NCDB database and evaluated patients who have nonmetastatic and potentially curable disease and proposed that patients undergo surgery following chemoradiation as definitive treatment, with the most favorable 5-year survival of patients with AA published to date [8]. Their results are similar to the earlier SEER database analysis made by Franklin et al. [8, 17], As reflected in treatment of patients with gastrointestinal adenocarcinoma, AA patients with complete surgical resection appear to have the best long-term survival interval [18].

This review is the largest review to date with regards to AA. Based on the review of the SEER database with long-term follow-up, several takeaway messages can be formulated. First, this disease has a bimodal distribution with younger and older patients having poor prognosis initially. Second, surgical intervention can improve outcome, with potential for longterm survival. Because this disease is an adenocarcinoma, surgical intervention should be the cornerstone of treatment with concurrent chemoradiation when appropriate. However, due to its location, the metastatic pattern of this tumor follows that of anal canal squamous carcinoma. Therefore, nodal metastasis to the groin nodes can occur early and should be part of the initial treatment planning [19].

Our study did have few limitations including being retrospective in nature. There is also a degree of heterogeneity in the reported dataset as the patients with adenocarcinoma of the anal canal is not a well-recognized clinical entity. Divisions into the zones of involvement, histological subtypes, and appropriate staging of anal canal adenocarcinomas are all areas of controversy which lacks definitive clarity. Moreover, the details about chemotherapy regimen in the reported series were not available. Nonetheless, given that the pathology is quite rare, this represents a large data series that will hopefully shed light on an otherwise unrecognized disease entity. Any definitive treatment plan 
should be formulated or recommended based on multidisciplinary treatment planning following disease presentation.

\section{Conclusion}

On the basis of our analysis of anal canal adenocarcinoma from the SEER database, it appears that the incidence of AA is decreasing. The prognosis of the disease remains dismal in the elderly. The bimodal distribution of poor short-term cancer-free survival in younger and older patients is also evident. Surgical resection improves the chance of cure over chemoradiation alone. We do feel the need for multicenter studies on AA given the lack of adequate research in this field.

\section{Data Availability}

The data supporting this SEER database analysis are available from the SEER database. All supplementary data are also included in the submitted manuscript. Alternatively, the data used to support the findings of this study are available from the corresponding author upon request.

\section{Conflicts of Interest}

The authors declare that they have no conflicts of interest.

\section{Supplementary Materials}

Supplementary Table S1: histology coding classification. Supplementary Table S2: tumor description. (Supplementary Materials)

\section{References}

[1] M. E. Abel, Y. S. Y. Chiu, T. R. Russell, and P. A. Volpe, "Adenocarcinoma of the anal glands," Diseases of the Colon \& Rectum, vol. 36, no. 4, pp. 383-387, 1993.

[2] R. J. Myerson, L. H. Karnell, and H. R. Menck, "The national cancer data base report on carcinoma of the anus," Cancer, vol. 80, no. 4, pp. 805-815, 1997.

[3] R. J. Kline, R. J. Spencer, and R. G. Harrison, "Carcinoma associated with fistula-in-ano," Archives of Surgery, vol. 89, no. 6, pp. 989-994, 1964.

[4] S. Anwar, H. Welbourn, J. Hill, and D. Sebag-Montefiore, "Adenocarcinoma of the anal canal-a systematic review," Colorectal Disease, vol. 15, no. 12, pp. 1481-1488, 2013.

[5] M. Herfs, P. Roncarati, B. Koopmansch et al., "A dualistic model of primary anal canal adenocarcinoma with distinct cellular origins, etiologies, inflammatory microenvironments and mutational signatures: implications for personalised medicine," British Journal of Cancer, vol. 118, no. 10, pp. 1302-1312, 2018.

[6] N. Esiashvili, J. Landry, and R. H. Matthews, "Carcinoma of the anus: strategies in management," The Oncologist, vol. 7, no. 3, pp. 188-199, 2002.

[7] D. L. Joon, M. W. T. Chao, S. Y. K. Ngan, M. L. Joon, and M. J. Guiney, "Primary adenocarcinoma of the anus: a retrospective analysis," International Journal of Radiation Oncology*Biology*Physics, vol. 45, no. 5, pp. 1199-1205, 1999.
[8] R. Li, A. Shinde, M. Fakih et al., "Impact of surgical resection on survival outcomes after chemoradiotherapy in anal adenocarcinoma," Journal of the National Comprehensive Cancer Network, vol. 17, no. 10, pp. 1203-1210, 2019.

[9] G. J. Chang, R. J. Gonzalez, J. M. Skibber, C. Eng, P. Das, and M. A. Rodriguez-Bigas, "A twenty-year experience with adenocarcinoma of the anal canal," Diseases of the Colon \& Rectum, vol. 52, no. 8, pp. 1375-1380, 2009.

[10] M. Basik, M. A. Rodriguez-Bigas, R. Penetrante, and N. J. Petrelli, "Prognosis and recurrence patterns of anal adenocarcinoma," The American Journal of Surgery, vol. 169, no. 2, pp. 233-237, 1995.

[11] E. Von Elm, D. G. Altman, M. Egger, S. J. Pocock, P. C. Gøtzsche, and J. P. Vandenbroucke, "The Strengthening the reporting of observational studies in epidemiology (STROBE) statement: guidelines for reporting observational studies," Journal of Clinical Epidemiology, vol. 61, no. 4, pp. 344-349, 2008.

[12] L. S. Jensen, M. H. Shokouh-Amiri, K. Hagen, H. Harling, and V. O. Nielsen, "Adenocarcinoma of the anal ducts. A series of 21 cases," Diseases of the Colon \& Rectum, vol. 31, no. 4, pp. 268-272, 1988.

[13] N. Malakhov, A. M. Kavi, A. Lee et al., "Patterns of care and comparison of outcomes between primary anal squamous cell carcinoma and anal adenocarcinoma," Diseases of the Colon \& Rectum, vol. 62, no. 12, pp. 1448-1457, 2019.

[14] M. Papagikos, C. H. Crane, J. Skibber et al., "Chemoradiation for adenocarcinoma of the anus," International Journal of Radiation Oncology*Biology*Physics, vol. 55, no. 3, pp. 669678, 2003.

[15] Y. Belkacémi, C. Berger, P. Poortmans et al., "Management of primary anal canal adenocarcinoma: a large retrospective study from the rare cancer network," International Journal of Radiation Oncology*Biology*Physics, vol. 56, no. 5, pp. 1274-1283, 2003.

[16] K. P. Beal, D. Wong, J. G. Guillem et al., "Primary adenocarcinoma of the anus treated with combined modality therapy," Diseases of the Colon \& Rectum, vol. 46, no. 10, pp. 1320-1324, 2003.

[17] R. A. Franklin, S. Giri, P. Valasareddy, L. T. Lands, and M. G. Martin, "Comparative survival of patients with anal adenocarcinoma, squamous cell carcinoma of the anus, and rectal adenocarcinoma," Clinical Colorectal Cancer, vol. 15, no. 1, pp. 47-53, 2016.

[18] N. Kounalakis, A. Artinyan, D. Smith, P. Mojica-Manoso, B. Paz, and L. L. Lai, "Abdominal perineal resection improves survival for nonmetastatic adenocarcinoma of the anal canal," Annals of Surgical Oncology, vol. 16, no. 5, pp. 1310-1315, 2009.

[19] J. Shia, "An update on tumors of the anal canal," Archives of Pathology and Laboratory Medicine, vol. 134, no. 134, pp. 1601-1611, 2010. 